\title{
Brand Building of Cultivating and Practicing the Socialist Core Values in Colleges and Universities From the Perspective of Internet Plus
}

\author{
Chen $\mathrm{Yu}^{1, \mathrm{a}}$, Wang Jiajia ${ }^{1, \mathrm{~b}}$ \\ ${ }^{1}$ Taizhou Polytechnic College, Jiangsu Taizhou, China, 225300 \\ axiaoyueryuchen@163.com bwangjiajia_99@163.com
}

Keywords: Internet plus, Core value cultivation, Organism, Brand building

\begin{abstract}
In the Internet plus era, constructing the core value cultivation organism is the internal demand of brand building of cultivating and practicing the socialist core values in colleges and universities, so we must pay close attention to the generative nature of the subject, the relevance of the individual and the coordination of the whole. Make supply and demand fit, classic and modern mix together, all kinds of resources integrate deeply.
\end{abstract}

\section{Introduction}

At present, the most typical problem existing in the cultivation and practice of socialist core values in colleges and universities is the deficiency of integrity and unity, the widespread existence of broken ways and the lack of close relationship between them, which makes it difficult to play a synergistic role effectively, especially in the Internet plus age. This kind of fragmentation noly has "quantity" but not "quality", the atomic type of cultivation, face the rotation impact of a variety of fragmentation of mass information, which is easy to dilute and dissipate quickly. We must strengthen the brand awareness of the Internet plus age, meticulously create some brands that has both fortress function and close connection one by one, and take these interrelated brands as the fundamental supporting points, fan out from a point to an area, link work at selected spots with that in entire areas. The fine grid of core value cultivation must be constructed in an all-round way so as to effectively cast a strong barrier against the impact of multiple values and a firm position to consolidate the core value position.

\section{Constructing the core value cultivation organism is the internal demand of brand building in the Internet plus age}

"The whole universe is an organic system, in which various events, and reality existence interrelate with each other and mutual inclusion. ", "the unit of composition of the universe is an organism consisting of properties and relationships. "[1] "Society today is not a solid crystal, but an organism that could change and is often in the process of change. "[2] The universe and society are "big organism" which are composed of concrete "small organisms" one by one. According to certain rules, all kinds of species and resources in nature can contract and complement each other. However, the cultivation and practice of core values sometimes has little effect and low efficiency, one of the most important reasons is the serious lack of organic property and the lack of close internal relations among the elements, so it is difficult to work together from the internal mechanism. In the past, because the technology level is not high, the production and exchange of information is neither rapid nor frequent, the organic lack of ideological and political work is not so obvious. But in the Internet plus age, the situation has completely different. The lack of organic nature makes the core value of cultivation fight each other and alone, and face the strong impact of multiple values, various interests and various temptations, many of its work is virtually empty.

With the acceleration of the Internet era, people's way of life and thinking will continue to produce profound changes, some changes will be revolutionary, subversive, if we can not build an organism in time, the cultivation of core values will gradually decline. Therefore, the cultivation and practice of socialist core values in colleges and universities, especially the brand building, must take the construction of organisms as the fundamental purpose, closely revolve around the intrinsic relevance, 
generative, developmental, dynamic and unceasing nature of the cultivation and practice of core values, each element has its own uniqueness and the relation with reality. In this process, the more obvious brand, the more perfect the organic features and the more comprehensive the function. At the same time, this is also an important way to effectively deal with the core value cultivation of flexibility, repetition, it can timely guide the direction, is the most effective measure to counter all kinds of interference online and offline, consolidate and strengthen the core value into the mind.

\section{The basic characteristics of brand building require a high degree of attention to cultivate the core value in the Internet plus age}

\subsection{The generative of subjects}

The cultivation and practice of core values are for real people, and there are two kinds of people in colleges and universities: faculty and young students. They are the responsible subjects of the cultivation and practice of the core values, the implementers of the core values, the creators of new behaviors and new ideas. The generative nature of organism emphasizes natural growth, but the process of natural growth has a certain order and must follow certain rules. The cultivation of core value is the same, the promotion of implementers and the acceptance of teachers, students and employees are also orderly. Each subject has its own differences, so it is necessary to strictly follow the scientific logic of cultivating core value and the growth law of all kinds of subjects. It pays full attention to the natural life state of each subject, individual subjective interest and will direction, synthetically gives policy, classifies the instruction, effectively guarantees the generation of all kinds of subjects, guides and standardizes their natural growth in an orderly manner.

\subsection{The relevance of individual}

Both Marx's social organism theory and Whitehead's organic philosophy always emphasize the intrinsic relevance of the process. As a basic method for Marxism to understand and transform the world, dialectical thinking requires that problems should be viewed in a conjunctive rather than in isolate, comprehensive rather than one-sided, changing rather than static way. Whitehead also believed that connectedness is the essence of everything that belongs to all types. In the process of cultivating and practicing the core value, we must pay close attention to the intrinsic relevance and coupling of these "elements" and "organism", and try to analyze and grasp the basic context, the pulse of the times and the inherent law from the complicated appearance of things. To implement measures, allocate resources scientifically and reasonable, so that each "small organisms" can not only play a corresponding function alone, but also can fit in with each other, and effectively realize the overall function of "large organism".

\subsection{The coordination of whole}

The cultivation and practice of core values is a complex system engineering. The system is a unified whole composed of several interrelated and interacting elements in a certain way. The function of the whole depends on the structure, that is, the way in which the elements are interrelated and interacted. Therefore, in the process of cultivating and practicing the core values, we should pay attention to the top-level design and overall planning, comprehensively strengthen the systematization, synergy and openness of various moves, and make sure that the structure is reasonable, the layers are clear, the nature is orderly, and the functions are perfect. So that the immediate and long-term coordination, local and overall matching, gradual and breakthrough link, we should also pay attention to the selection of important areas and joint links as a breakthrough point, move the whole body on the key points, carefully build the core value to cultivate the brand, and give full play to its supporting point and connection point, so that the initiatives complement each other, promote each other and benefit by associating together. 


\section{The thinking mode and path choice of brand building of cultivating the core value int the Internet plus age}

Based on the above analysis, the brand building of the core value of the Internet plus age should be based on two points at least: one is the Internet thinking, and the two is the construction of the organism. Only to spread out from these two dimensions, find the right positioning, accurate power, it can play a multiplier effect.

\subsection{Respecting the status of the subject and making the supply and demand fit appropriately}

Due to the lack of full respect for the principal position of teachers and students, lack of sufficient attention to their psychological experience, the large number of big pot rice, the small number of buffet meals, to be more serious than to be lively, which lead to a mismatch between supply and demand, or even a serious imbalance between supply and demand. This makes ome teachers and students feel boring about core values education. To change this situation, we must deeply realize that teachers and students in colleges and universities, especially the students who constitute the vast majority, are flesh and blood, sentimental individuals, and, as far as possible, provide diversified choices that can meet their individual needs and individual development. Attract them to participate actively and feel great pleasure in them, thus stimulate the endogenous motivation of active learning and practice, ensure the healthy and orderly growth of core values in them. Here, we can make full use of the advantages of the convenient communication on the Internet, and timely feedback the individual's trivial, scattered, superficial and even secular psychological experience to the specific implementer, and be summed up by the implementer. Timely interactive communication, effective guidance after deep thinking, the effect will naturally be different. To achieve this goal, not only through the traditional classroom, community activities, but also through instant messaging platform, eg WeChat QQ and so on, has a strong operability.

\subsection{Breaking the limitation of thinking and integrating classical modern highly}

The essence of Internet thinking is to combine Internet platform with other things, the fundamental aim is to cross and merge. The cultivation of the core value is the same, it is necessary to give full play to the role of all kinds of ways below the line, and to make good use of network, which is a novel and fashionable element, to further enlarge the functions of other ways. General Secretary Xi Jinping stressed "We should promote the high integration of traditional advantages of ideological and political work with information technology and enhance the sense of the times and attractiveness." In this respect, the most valuable and typical significance, should belong to the excellent Chinese traditional culture gets a new lease of life. Needless to say, the ancient Chinese traditional culture has gradually gone to death because of its inflexibility and feudalism. It is the integration of Marxism that reactivates the excellent factors among them and gives rise to vitality. Xi Jinping General Secretary praised as "the foundation of a firm foothold in the world's cultural turmoil." Today, how to make good use of these contents is a new and important subject in front of us. China Central Television has made a good demonstration, the Chinese poetry Congress, idiom assembly, drama Congress, riddle assembly, Chinese dictation Congress and other excellent traditional cultural programs, which are integrated with modern fashion elements, are popular and highly praised by Chinese people. We should get profound inspiration from it, make good use of all kinds of modern technology, combine with our own reality to innovate energetically, make the traditional classics radiate again, and strongly promote the cultivation and practice of the core value of colleges and universities.

\subsection{Adjusting the structure of the elements and integrating all kinds of resources highly}

The main reason for the lack of organic in the previous cultivation mode is that the distribution of each element is disorderly and scattered, can not form an integrated force. In order to change this situation, it is necessary to combine the elements according to certain theoretical logic and practical logic, which can not only achieve the division of work and cooperation, but also realize the function complementation, and ensure that the cultivation goal of core value can be realized to the maximum extent. At present, the reason why the cultivation of core value in many colleges and universities is 
inefficient lies in the unreasonable and uncoordinated cultivation structure, that is, the imbalance of internal and external factors of organic elements of core value cultivation.

Therefore, the cultivation of brand, we must adjust the structure of the elements as the main direction of attack. In line with the basic principle of technology is first, direction for want, content as king, and effectiveness as the basis, and in accordance with the learning, work and life realities of teachers, students and staff, we should deeply analyze the profound impact of all elements, including the Internet, on them. Having a comprehensive understanding of their common characteristics and individual needs, and effectively integrating the development and use of all types of network resources, internal and external resources, and the rational allocation of the proportion of these elements, in accordance with their affinity and influence. So that classroom education, extracurricular practice, network influence and so on can be re-sequentially optimized and strengthened under the control and cohesion of the core objectives, and their functions mutual excitation, which can fully reflect the cohesion and energetic of the system, and fully highlight the single action and diffusive force, especially the powerful force and diffusive force of Weibo, WeChat, micro-video and other Internet products. It makes solos into ensemble, each element can play its own cultivating function effectively, and can converge into a continuous sonata.

\section{Acknowledgement}

This research was financially supported by the Special Project on ideological and political work of philosophy and Social Sciences Research in Jiangsu Province(2017SJBFDY683), and the Key projects of Scientific Research Foundation of Taizhou Vocational and Technical College(TZYKYZD-17-4), and the Funded projects of "six groups" of talents engineering training object in propaganda culture system in Taizhou City.

\section{References}

[1] Whitehead. Study on process and reality-cosmology (preface)[M], Yang Fubin, translation. Beijing: Renmin University of China press, 2013:24.

[2] Marx, Engels. Selected works of Marx and Engels: volume 2[M]. Beijing: People's Publishing House, 1995:102. 